\title{
INTERthesis
}

DOUTORADO INTERDISCIPLINAR EM CIÊNCIAS HUMANAS - UFSC - FLORIANÓPOLIS - SC - BRASIL

\section{PERCEPÇÃO DE DIFERENÇAS INTERGRUPAIS E INFRA-HUMANIZAÇÃO}

\section{Resumo:}

\section{Sheyla Christine Santos Fernandes ${ }^{1}$ Marcos Emanoel Pereira ${ }^{2}$}

Este estudo teve como objetivo analisar a atribuição diferenciada de emoções e traços a três alvos (Brasileiros, brancos e negros) e a partir dos achados discutir alguns aspectos teóricos e metodológicos da infra-humanização dos grupos sociais. Participaram do estudo 164 estudantes universitários de Maceió, Alagoas, com idades entre 18 e 41 anos (Média $=20,64$; DP $=3,88$ ). Os estudantes responderam às escalas de atribuição diferenciada de emoções (PALADINO ET AL., 2000) e de traços (MOSCOVICl \& PEREZ, 1999). Os principais resultados endossam parcialmente a infra-humanização dos negros e o favorecimento endogrupal ou do grupo majoritário. As peculiares da infra-humanização no contexto desta pesquisa são discutidas através do papel das normas sociais no estudo de atitudes antinormativas.

Palavras-chave: Percepção. Atitudes. Racismo. Normas Sociais. InfraHumanização.

\section{PERCEPTION OF INTERGROUP DIFFERENCES AND INFRA-HUMANIZATION}

\section{Abstract:}

The aim of this study was to analyze the differentiated attribution of emotions and traits to three groups (Brazilian, white and black) and from the findings discuss some theoretical and methodological aspects of the infra-humanization of social groups. A total of 164 university students from the city of Maceió, aged 18-41 years (mean = 20.64, SD $=3.88$ ) participated in the study. The students responded to the scales of differentiated attribution of emotions (PALADINO ET AL., 2000) and traits (MOSCOVICI \& PEREZ, 1999). The main results partially indicate the infrahumanization of black people and the ingroup favoring or the majority group. The peculiarities of infra-humanization in the context of this research are discussed through the role of social norms in the study of anti-normative attitudes.

Keywords: Perception. Attitudes. Racism. Social Norms. Infra-Humanization.

\section{PERCEPCIÓN DE DIFERENCIAS INTERGRUPALES E INFRA-HUMANIZACIÓN}

\section{Resumen:}

\footnotetext{
1 Doutora em Psicologia Social pela Universidade Federal da Bahia. Professora do curso de PósGraduação e Graduação em psicologia na Universidade Federal de Alagoas, Maceió, AL, Brasil Email: sheyla.fernandes@ip.ufal.br

2 Doutor em Psicologia Social pela Universidade Federal do Rio de Janeiro. Pós-doutorado na Universidade Complutense de Madrid. Professora do curso de Pós-graduação e Graduação em psicologia da Universidade Federal da Bahia, Salvador, BA, Brasil E-mail: memanoel@gmail.com
} 
Este estudio tuvo como objetivo analizar la atribución diferenciada de emociones y rasgos a tres grupos (Brasileños, blancos y negros) y a partir de los hallazgos discutir algunos aspectos teóricos y metodológicos de la infra-humanización de los grupos sociales. En el estudio participaron 164 estudiantes universitarios de Maceió, Alagoas, con edades entre 18 y 41 años (Media $=20,64$, DP $=3,88$ ). Los estudiantes respondieron a las escalas de atribución diferenciada de emociones (PALADINO ET AL., 2000) y de rasgos (MOSCOVICI \& PEREZ, 1999). Los principales resultados respaldan parcialmente la infra-humanización de los negros y el favorecimiento endogrupal o del grupo mayoritario. Las peculiares de la infrahumanización en el contexto de esta investigación son discutidas a través del papel de las normas sociales en el estudio de actitudes antinormativas.

Palabras clave: Percepción. Actitudes. Racismo. Normas Sociales. Infrahumanización.

\section{INTRODUÇÃO}

O interesse em estudar a percepção de diferenças intergrupais em uma perspectiva psicossociológica é remetido à data relativamente próxima à fundação da disciplina de psicologia social. Alguns autores indicam que por volta de 1930 foi publicado o primeiro estudo que considerou empiricamente esta temática (HASLAM, JETTEN, POSTMES \& HASLAM, 2009), referindo-se às análises realizadas por Katz e Braly (1933) sobre a origem dos estereótipos em relação a grupos étnicos e nacionalidades.

Katz e Braly (1933) construíram uma lista com 84 atributos dirigidos a dez grupos distintos (alemães, americanos, ingleses, chineses, italianos, irlandeses, turcos, japoneses, judeus e negros), requisitando dos participantes do estudo que selecionassem os traços mais típicos para os caracterizar. Os resultados mais evidentes associaram os alemães aos atributos industriosos, ponderados, metódicos; os italianos, aos atributos passionais, artísticos, impulsivos; os negros foram caracterizados como supersticiosos, preguiçosos, ignorantes; as qualidades agressivas dos judeus foram enfatizadas; aos americanos foram atribuídas características como materialistas, progressistas, inteligentes, dentre outros. $\mathrm{O}$ alto nível de concordância entre os participantes é o dado que mais chamou a atenção nesse estudo. Para os autores, as pessoas não julgam os grupos com base em verdades, mas com base no que é socialmente disseminado, expressando inclusive, um elevado índice de preconceito.

Em termos cognitivos, os estereótipos simplificam a configuração das informações sociais (TAJFEL, 1981), agilizando o processo de categorização social e tomada de decisão, contudo, essa ativação tem potencial consequência danosa às 
relações sociais (CABECINHAS, 2002), uma vez que as particularidades dos grupos e das pessoas são excluídas, sendo o processo de homogeinização automaticamente acionado.

É reportado ao estudo de Taylor et al. (1978) a primeira explicação para os efeitos da homogeinização dos grupos externos ao percebedor (exogrupos). De acordo com seus achados, a percepção de diferenças estaria associada à familiaridade e a percepção de homogeneidade, à não familiaridade. Enquanto a pessoa avalia o próprio grupo (endogrupo) mais cuidadosamente, percebendo maior variabilidade dos membros, o exogrupo é percebido de forma mais homogênea, gerando a imputação dos estereótipos e preconceitos.

Nas últimas décadas os estudos sobre julgamento social e percepção de diferenças entre grupos têm sido realizados sob diversas perspectivas (RUTLAND \& KILLEN., 2015; ET AL., 2014; LEYENS \& VALA, 2016). No presente trabalho, duas delas serão discutidas, (1) atribuição diferenciada de emoções e (2) atribuição diferenciada de traços.

\section{ATRIBUIÇÃO DIFERENCIADA DE EMOÇÕES}

De acordo com Leyens et al. (2000), o julgamento social pode ser apreendido através da atribuição de emoções humanas e não-humanas às categorias sociais. Existe uma propensão para avaliar o endogrupo como sendo mais humano do que outros, no entanto, essa propensão não necessariamente ocorre nos casos em que existe hostilidade intergrupal. De fato, as relações endogrupo vs. exogrupos são colocadas como importantes preditores do julgamento social (MUSSWEILER, 2003), o que implica na ideia de que as pessoas estão inclinadas a experienciar este processo de forma tendenciosa por meio do favoritismo endogrupal ou etnocentrismo, e a consequência deste processo seria uma percepção tendenciosa do "nós" em relação ao "eles" (GAUNT, LEYENS \& DEMOULIN, 2002; DERKS ET AL., 2014).

Alguns estudos demonstram que as pessoas analisam com maior cuidado um comportamento ambíguo de um membro do próprio grupo em detrimento da análise do comportamento semelhante de um membro de outro grupo (DASGUPTA, 2004; 2009; DELGADO ET AL., 2012), assim como, estão propensas a desculpar um comportamento desviante de um membro do endogrupo em detrimento do comportamento antinormativo do membro do exogrupo (EIDELMAN ET AL., 2006; 
BETTENCOURT ET AL., 2016). Tal tendenciosidade é evidenciada em um conjunto de análises (DESCHAMPS ET AL., 2005; HARVEY ET AL., 2017) e segue desde uma avaliação mais positiva do endogrupo a uma avaliação mais negativa do exogrupo, mesmo na ausência de comprovações ou na presença de fatos imprecisos. Mais do que simplesmente desqualificar o exogrupo ferindo sua imagem, esta inclinação possui a função de proteção da auto-imagem grupal (YZERBYT ET AL., 2000; VALA ET AL., 2015), além de contribuir para o aumento da coesão e cooperação no interior do grupo (HAMMOND \& AXELROD, 2006; RUTLAND ET AL., 2015).

Por outro lado, tendo em vista que o julgamento baseado no favoritismo endogrupal pode endossar atitudes e comportamentos negativos em relação ao exogrupo, ao avaliar o endogrupo como mais humano do que outros, o indivíduo estaria guiando seu julgamento a partir do favoritismo endogrupal e a consequência deste processo seria a infra-humanização dos grupos externos (LEYENS ET AL., 2000).

Estudos realizados em diferentes nações (ver GAUNT ET AL., 2002; HU ET AL., 2017; MARTíNEZ ET AL., 2017) têm demonstrado que algumas emoções são percebidas como atributos compartilhados por humanos e outras espécies, porém, determinados tipos de emoções são percebidas unicamente como atributos humanos. Por exemplo, emoções como medo, raiva ou surpresa podem ser experimentadas algumas vezes por animais, sendo denominadas de emoções primárias, mas emoções como esperança, admiração ou arrependimento, são experimentadas apenas por seres humanos, sendo então denominadas emoções secundárias. Emoções primárias possuem bases biológicas e podem ser experimentadas por outros primatas, são caracterizadas pela fugacidade e sua ocorrência é explícita, enquanto que as emoções secundárias são resultantes da aprendizagem social (LEYENS ET AL., 2001).

Paladino et al. (2002) testaram o favoritismo endogrupal utilizando o Implicit Association Task (IAT). Os pesquisadores avaliaram que emoções são consideradas exclusivamente humanas e como podem ser associadas a endogrupos e exogrupos. Os resultados apresentaram fortes associações entre endogrupo e emoções secundárias e exogrupos e emoções primárias. A lista que segue no Quadro 1 apresenta o conjunto de emoções utilizado como estímulo nos estudos realizados pelos autores. 
Quadro 1: Lista de emoções primárias e secundárias (PALADINO ET AL., 2002)

\begin{tabular}{|lll|}
\hline Valência & Emoções Primárias & Emoções Secundárias \\
\hline & Desejo & Despeito \\
& Luxúria & Decepção \\
Positivas & Atração & Culpa \\
& Surpresa & Remorso \\
& Prazer & Vergonha \\
& Alegria & Espanto \\
& Excitação & \\
\hline Crueldade & Admiração \\
& Medo & Compaixão \\
& Dor & Felicidade \\
Negativas & Terror & Empatia \\
& Raiva & Afeto \\
& & Serenidade \\
& & Amizade \\
& & Esperança \\
& & Amor \\
\hline
\end{tabular}

Emoções são inferidas e interpretadas pelo percebedor e por esta característica particular são fortemente associadas a percepções enviesadas. Por exemplo, os atributos inteligência e linguagem podem ser facilmente associados a pessoas bem-educadas e de alto padrão social. No entanto, com as emoções o processo de julgamento social é diferente. Um estudo realizado por Fiske et al. (1999), demonstrou que a atribuição de emoções ocorre independente do status do grupo percebido. Os pesquisadores encontraram que um grupo de alto status na maior parte dos casos é percebido como competente mesmo que as pessoas não gostem dele, porém, um grupo de baixo status, na mesma proporção, é percebido como incompetente, mesmo que as pessoas o considerem agradável.

Outras evidências apontam que a percepção de emoções secundárias induz comportamentos solidários (BATSON ET AL., 2007; WONDRA \& ELLSWORTH, 2015), do mesmo modo que refletir sobre as experiências emocionais das pessoas pode gerar sentimentos de empatia (BATSON, 2009; BARASCH ET AL., 2014).

Em comum essas evidências demonstram que a atribuição de emoções pode ser um adequado termômetro de infra-humanização e, ao passo que está associada à inferência de outras emoções, apresenta ser um possível preditor de crenças, atitudes e escolhas comportamentais.

\section{ATRIBUIÇÃO DIFERENCIADA DE TRAÇOS}

Outra forma de analisar a percepção de diferenças entre os grupos é através da atribuição diferenciada de traços naturais e traços culturais (VILHENA, 2007; BAIN, 2014). Os traços naturais são definidos como atributos utilizados para referir 
humanos e animais e os traços culturais são exclusivamente utilizados para referir seres humanos (MOSCOVICI \& PÉREZ, 1999). As características indicadas como exclusivamente humanas são flexíveis ao contexto e são aprendidas por meio do processo de interação. As características utilizadas para descrever seres humanos e animais são rígidas, geralmente de ordem biológica e inatas (Quadro 2).

Quadro 2: Dimensões do conteúdo dos traços naturais e culturais em função da valência (AGUIAR \& LIMA, 2001)

\begin{tabular}{|lll|}
\hline Valência & Traços Naturais & Traços Culturais \\
\hline & Esperto & Sincero ou leal \\
& Alegre & Inteligente \\
Intuitivo & Progressista \\
& Espontâneo & Sábio \\
& Fisicamente hábil & Competente \\
& Livre & Industrioso \\
& Dócil & Civilizado \\
& Simples & \\
& & \\
\hline & Agressivo & \\
& Ruidoso & Falso ou mentiroso \\
& Selvagem & Infantil \\
& Impulsivo & Supersticiosos \\
& Descontrolado & Conservador \\
& Estúpido & Materialista \\
& Feroz & Desonesto \\
& & \\
& & \\
& & \\
& & \\
& & \\
& &
\end{tabular}

$\mathrm{Na}$ medida em que certa categoria social é mais associada a traços naturais do que traços culturais, infere-se que sua condição de humanidade é inferior a uma categoria social que possua mais traços culturais. Moscovici e Pérez (1997) desenvolveram um estudo junto a espanhóis com o objetivo de avaliar de que forma a adesão à cultura dominante por parte do exogrupo (neste caso, os ciganos) pode afetar a percepção que o endogrupo constrói do exogrupo. Os participantes foram convidados a ler um texto acerca da experiência dos ciganos na Espanha em dois contextos distintos, (1) os ciganos não aderiam à cultura dominante e (2) os ciganos aderiam à cultura dominante. Utilizando uma lista de adjetivos positivos e negativos que refletem traços naturais e culturais, os participantes emitiram seu julgamento. Foi possível concluir que os ciganos que não aceitam a integração cultural foram percebidos pelos espanhóis através de atributos mais naturais (por exemplo, intuitivo, fisicamente hábil, selvagem) do que culturais (por exemplo, criativo, leal a sua identidade, perverso, mal-intencionado), por outro lado, os ciganos que permitiram a integração cultural receberam uma quantidade inferior dos atributos naturais. 
Análises subsequentes investigando o processo de infra-humanização a partir da atribuição de traços culturais e naturais (LIMA \& VALA, 2005; VALA, 2013) encontraram resultados consistentes com os de Moscovici e Pérez, o que indica que a atribuição de traços naturais ou a negação de traços culturais a determinadas categorias sociais é um potente catalisador da saliência das diferenças intergrupais que confere a determinados grupos o estatuto de menos humanos do que outros ou de infra-humanizados.

Deschamps et al. (2005) testaram a hipótese de que o racismo e a xenofobia se expressam por meio da atribuição diferenciada de traços ao endogrupo (suíços) e aos exogrupos (negros africanos e mulçumanos). Os resultados apresentaram a associação entre traços culturais e o endogrupo e traços naturais e os exogrupos. Os autores apontaram a existência do favoritismo endogrupal e da rejeição exogrupal no processo de atribuição de traços, contudo é importante ressaltar que na percepção de exogrupos que possuem o mesmo estatuto social este fenômeno não se apresenta desta forma. Em relação aos grupos de mesmo nível social e avaliados como pertencentes às mesmas origens do endogrupo, os participantes não atribuem os traços naturais.

Costa-Lopes e seus colegas (2008) referem que os processos de percepção e julgamento social permitem a construção de conhecimento sobre nós mesmos, sobre os outros, sobre os grupos em que nos sentimos pertencentes e sobre os grupos que rejeitamos. Desta forma, as maneiras pelas quais elaboramos a percepção e o julgamento intergrupal operam segundo a lógica dos repertórios originados das relações estabelecidas entre o "nós" e os "eles", de modo que o sentido e o valor atribuído às semelhanças e às diferenças se configuram como aspectos centrais na construção dos processos inerentes às relações intergrupais. Diante do exposto, o presente estudo tem a finalidade de investigar a percepção de diferenças intergrupais por meio da atribuição de diferenciada de emoções e traços a três grupos, brasileiros, brancos e negros. Espera-se que ao alvo "brasileiros", por representar o endogrupo dos respondentes e ao alvo "brancos", que constitui o grupo majoritário, não sejam dirigidos atributos infra-humanizadores. Por outro lado, em relação ao alvo "negros", estima-se a infra-humanização por meio da negação de atributos superiores ou por meio da atribuição de emoções e traços inferiores. 


\section{MÉTODO}

Participaram do estudo 164 estudantes universitários de duas universidades da cidade de Maceió, Alagoas, sendo a maioria do sexo feminino $(61,1 \%)$, solteiros $(94,3 \%)$, católicos $(60 \%)$, auto-categorizados como não-brancos $(61,7 \%)$ e com idades entre 18 e 41 anos (Média = 20,64; Desvio Padrão = 3,88).

Os estudantes responderam a dois instrumentos, além de questões sociodemográficas:

(1) Escala de expressão de sentimentos e emoções baseada no estudo de Paladino et al. (2002) para medir a infra-humanização. Utilizamos um modelo composto por oito emoções, sendo quatro emoções primárias - duas positivas (contentamento e excitação) e duas negativas (raiva e irritação) - e quatro emoções secundárias - duas positivas (compaixão e esperança) e duas negativas (amargura e tristeza). Este modelo de oito emoções foi utilizado por Vala et al. (2009) e, com base na sugestão de Castano e Giner-Sorolla (2006), determina que a infrahumanização ocorre quando os resultados apontam diferenças entre as atribuições de emoções ao endogrupo e o exogrupo. Desta forma, os participantes foram requeridos a responder a frequência com que três grupos (brasileiros, negros e brancos) expressavam os sentimentos e emoções listados. Para responder ao instrumento, foi utilizada uma escala de respostas do tipo Likert de cinco pontos, sendo 1 referente a "nunca expressam" tais emoções e 5 referente a "sempre expressam".

(2) Escala de atribuição de traços naturais e culturais. Esta escala avalia a infra-humanização dos grupos no nível da atribuição de traços (MOSCOVICl \& PEREZ, 1999). Uma validação da diferenciação de atribuição de traços no contexto do Brasil foi realizada por Lima e Vala (2004), resultando em uma lista de doze características ou traços. Seguindo tais estudos, utilizamos uma lista mais parcimoniosa apresentada por Vala et al. (2009) em um estudo realizado em Portugal. Desta forma, a lista utilizada por este trabalho consistiu na apresentação de oito traços, sendo quatro traços de natureza (dócil, intuitivo, espontâneo e simples) e quatro de cultura (criativo, inteligente, civilizado e honesto). Neste contexto, os participantes foram requeridos a indicar a extensão em que cada traço é típico de brasileiros, negros e brancos em uma escala de respostas do tipo Likert de 5 pontos, sendo 1, "nada típico" a 5 "totalmente típico". A diferenciação de 
atribuição de traços naturais e culturais ao endogrupo em relação ao exogrupo é o que determina a infra-humanização, sendo constatada pela menor atribuição de traços de cultura ao exogrupo.

Os estudantes foram convidados a participar do estudo na situação de sala de aula, onde, após o convite e as devidas explicações, preenchiam um termo de consentimento livre e esclarecido a fim de assegurar seus direitos com base nas diretrizes das Resoluções 196/96 e 304/2000 do Conselho Nacional de Saúde acerca da ética em pesquisa com seres humanos, assinavam concordando com a participação e tomavam parte na pesquisa.

\section{RESULTADOS}

Inicialmente os indicadores das emoções primárias e secundárias, bem como, os indicadores dos traços naturais e culturais foram criados, somando-se as emoções e os traços, respectivamente.

Realizamos testes $t$ pareados para analisar se existiam diferenças entre as atribuições de emoções em função dos alvos (brasileiros, negros e brancos). Três hipóteses foram elaboradas para esse conjunto de resultados: (1) o alvo "brasileiros", que constitui o endogrupo deste estudo, receberia menos indicações de emoções primárias que os demais alvos, (2) o alvo "negros", que constitui o grupo minoritário, receberia mais emoções primárias em detrimento dos alvos brasileiros e brancos e (3) o alvo "brancos" receberia menos emoções primárias que o alvo negro. Os resultados demonstraram que não há diferenças na atribuição de emoções primárias aos três alvos. Para os participantes, brasileiros, negros e brancos experienciam emoções primárias de forma semelhante (Tabela 1).

Tabela 1: Dados descritivos da atribuição de emoções primárias

\begin{tabular}{llll}
\hline Emoções primárias & Brasileiros & Negros & Brancos \\
\hline Média & 2,90 & 2,91 & 2,95 \\
DP & 0,46 & 0,46 & 0,41 \\
EP & 0,37 & 0,37 & 0,33 \\
IC & 2,83 & 2,84 & 2,89 \\
\hline \multicolumn{4}{r}{ Nota: DP = Desvio padrão; EP = Erro padrão; IC $=$ Intervalo de confiança }
\end{tabular}

Em relação às emoções secundárias, levantamos as seguintes hipóteses: (1) o alvo "brasileiros" receberá mais indicações de emoções secundárias em detrimento dos demais alvos, corroborando com a tese do favoritismo endogrupal, (2) ao alvo "negros" será negada a atribuição de emoções secundárias em comparação aos alvos "brasileiros" e "brancos" e (3) o alvo "brancos" será favorecido 
em relação ao alvo "negros", recebendo a atribuição de mais emoções secundárias. Os resultados confirmaram as hipóteses parcialmente (Tabela 2). Os estudantes indicaram que os brancos experienciam com maior frequência emoções secundárias ( édia $=2,72)$ em comparação com os negros (média $=2,64)(t=2,693 ; p<0,05)$. Não foram encontradas diferenças significativas entre a atribuição de emoções secundárias indicada para os brasileiros e os demais grupos.

Tabela 2: Dados descritivos da atribuição de emoções secundárias

\begin{tabular}{llll}
\hline Emoções secundárias & Brasileiros & Negros & Brancos \\
\hline Média & 2,64 & 2,65 & 2,73 \\
DP & 0,48 & 0,46 & 0,46 \\
EP & 0,38 & 0,36 & 0,36 \\
IC & 2,56 & 2,58 & 2,66 \\
\hline
\end{tabular}

Nota: $\mathrm{DP}=$ Desvio padrão; EP = Erro padrão; IC = Intervalo de confiança

Para analisar a atribuição diferenciada de traços em função dos alvos, foram realizados os mesmos testes estatísticos (testes $t$ pareados). No que se refere à atribuição diferenciada de traços naturais, elencamos três hipóteses: (1) aos brasileiros será atribuída uma quantidade inferior de traços naturais, (2) uma quantidade maior de traços naturais será atribuída aos negros e (3) os brancos receberão uma quantidade menor de atribuição de traços naturais em relação aos negros. Os resultados indicaram não haver diferenças estatisticamente significativas entre as atribuições de traços naturais (Tabela 3 ).

Tabela 3: Dados descritivos da atribuição de traços de natureza

\begin{tabular}{llll}
\hline Traços de natureza & Brasileiros & Negros & Brancos \\
\hline Média & 2,43 & 2,35 & 2,60 \\
DP & 0,60 & 0,57 & 0,58 \\
EP & 0,47 & 0,45 & 0,46 \\
IC & 2,35 & 2,26 & 2,61 \\
\hline
\end{tabular}

Nota: DP = Desvio padrão; EP = Erro padrão; IC = Intervalo de confiança

Em relação aos traços de cultura, as hipóteses elaboradas indicavam que (1) os brasileiros receberiam mais atribuições de traços de cultura, (2) aos negros seriam recusados traços de cultura e (3) os brancos receberiam mais atribuição de traços culturais em detrimento do alvo "negros". Algumas hipóteses foram confirmadas. Mais traços de cultura foram indicados aos brasileiros (média $=2,49$ ) que aos negros (média $=2,41)(t=3,006 ; p<0,005)$. Os traços de cultura atribuídos aos brancos e negros não apresentaram diferenças significativas (Tabela 4).

Tabela 4: dados descritivos da atribuição de traços de cultura

\begin{tabular}{llll}
\hline Traços de cultura & Brasileiros & Negros & Brancos \\
\hline Média & 2,49 & 2,41 & 2,43 \\
DP & 0,53 & 0,56 & 0,52 \\
EP & 0,41 & 0,44 & 0,41 \\
IC & 2,49 & 2,33 & 2,35 \\
\hline
\end{tabular}


Nota: $\mathrm{DP}=$ Desvio padrão; $\mathrm{EP}=$ Erro padrão; IC = Intervalo de confiança

\section{DISCUSSÃO}

Objetivando analisar a atribuição diferenciada de emoções e traços a três alvos e a partir dos achados discutir alguns aspectos teóricos e metodológicos da infra-humanização, o presente estudo foi desenvolvido. Em termos gerais, verificamos sobre a infra-humanização que nem todos os indicadores se apresentaram consistentes com outras investigações (PALADINO ET AL., 2002; LIMA, 2003). Esperávamos que o alvo "negros" recebesse mais emoções primárias e traços de natureza do que os alvos "brasileiros" e "brancos", assim como que os participantes atribuíssem mais emoções secundárias e traços de cultura aos brasileiros e, secundariamente, aos brancos. Nossas hipóteses foram confirmadas parcialmente. Verificamos que apenas a atribuição de emoções secundárias apresenta diferença significativa em função dos alvos "brancos" e "negros": brancos receberam mais emoções secundarias que negros. Em relação à atribuição de traços naturais, os resultados indicaram associações semelhantes entre os traços e os alvos, indicando que os três grupos são qualificados de maneira semelhante. Sobre a atribuição de traços de cultura, os resultados foram consistentes com o esperado: os brasileiros receberam mais traços de cultura que os negros. Em relação aos brancos não foram encontradas diferenças significativas.

Em resumo, para a infra-humanização via atribuição diferenciada de emoções, os resultados não foram consistentes com a tese do favoritismo endogrupal (DERKS ET AL., 2014; LEYENS ET AL., 2000), posto que os participantes não favoreceram o alvo "brasileiros". Porém o alvo pertencente ao grupo majoritário (brancos) foi favorecido em detrimento do alvo do grupo minoritário (negros). Em se tratando da infra-humanização via atribuição de traços, apenas em uma das comparações a tese do favoritismo endogrupal foi consistente, em relação à atribuição de traços de cultura aos brasileiros.

Segundo Cabecinhas (2002), que encontrou resultados semelhantes aos nossos, a ausência de manifestação de favoritismo endogrupal pode estar ligada a preocupação de não expressar preconceito e deste modo não ferir as normas antiracistas. Por outro lado, a autora refere que pode também estar ligada ao fato de os participantes terem respondido suas atribuições em escalas independentes, tendo com isto a possibilidade de não negar traços de ordem superior ao outro grupo pelo 
simples fato de terem atribuído tais traços ao próprio grupo. Cabecinhas, pôs a prova sua hipótese testando outro modelo de apresentação da medida de atribuição, desta vez, utilizando uma escala interdependente (variando de muito típico do próprio grupo a muito típico do exogrupo para cada adjetivo). Cabe ressaltar que este estudo foi realizado em Portugal com participantes angolanos e portugueses. Seus resultados indicaram que a mudança de estratégia de coleta de dados provocou uma mudança significativa nos resultados. Com o modelo de coleta de dados de escalas independentes, os resultados demonstraram que avaliações endogrupais são ligadas ao favoritismo apenas para os angolanos, mas não para os portugueses, mas com o segundo modelo, interdependente, tanto atribuições positivas quanto negativas são direcionadas aos dois grupos. Em nosso estudo, o modelo de instrumento utilizado permitia a visualização dos três alvos simultaneamente, o que se assemelha ao modelo interdependente utilizado por Cabecinhas na medida em que possibilita comparação das atribuições.

Sugerimos que a utilização de escalas interdependentes, como foi o caso de nossa investigação, comparando as atribuições intergrupais, pode interferir na resposta dos participantes, que para seguirem a norma do "politicamente correto" realizam atribuições balanceadas. Conforme Cabecinhas (2002), acreditamos que esse modelo torna saliente a norma social anti-racista e promove respostas conscientes voltadas para o igualitarismo.

Para além da metodologia empregada ativar normas sociais anti-racismo, cabe enfatizar a natureza das relações raciais brasileiras como possível dado a justificar os resultados obtidos em nossa investigação. A mistura de raças consiste na marca central da identidade brasileira (FERNANDES, 2011), mesmo que isso não implique em relações raciais justas, diverge amplamente da dinâmica de relações raciais características de outras nações. Logo, modelos teórico-metodológicos adaptados para o Brasil para estudar as relações raciais podem implicar em algumas lacunas.

Com efeito, o preconceito contra negros no Brasil parece se associar diretamente à cor da pele das pessoas, enquanto que em outras nações, o preconceito tem base na origem e se fundamenta na suposição de que o indivíduo descende de um grupo racial desfavorecido, portanto mesmo sem apresentar traços aparentes, o preconceito e sua expressão acontecem da mesma forma. Isso demonstra que estudar a base das diferenças raciais no Brasil é tarefa complexa e 
que se coloca imperativa na agenda de pesquisa sobre as relações raciais nesse contexto.

Lima e Vala $(2004 ;$ 2005), demonstram que a expressão do racismo é bastante sensível ao contexto sociocultural e, mais especificamente, ao contexto imediato de resposta. Em seus achados, foi possível perceber que os negros são infra-humanizados na medida em que o fracasso aparece mais associado a eles que aos brancos. Porém, quando os negros obtêm sucesso, menos atributos infrahumanizadores são aplicados a eles. Os autores acrescentam ainda que os brasileiros tendem a apresentar um elevado cuidado com a auto-imagem de pessoas justas e igualitárias no que se refere à questão do racismo e isso pode implicar em mais um obstáculo nos estudos acerca dessa temática.

\section{CONSIDERAÇÕES FINAIS}

O presente estudo evidencia alguns elementos que podem ser discutidos em nível teórico e metodológico sobre as relações intergrupais em geral e sobre as relações raciais em particular. Foi possível observar que a infra-humanização, enquanto construto indicativo de preconceito/racismo, em nosso contexto não apresenta distinções claras nas atribuições diferenciadas de emoções e traços aos alvos sociais aqui investigados. Associações consistentemente encontradas em diferentes nações entre infra-humanização e grupos minoritários não foram evidenciadas em nosso estudo, da mesma forma não foi possível corroborar com a tese do favoritismo endogrupal tal como esperado. Tivemos as hipóteses confirmadas parcialmente. Sugerimos que tanto as normas sociais e raciais pertinentes ao contexto brasileiro, como o formato empregado na aplicação do instrumento tenham gerado um efeito importante na expressão das respostas dos participantes.

Reiteramos a necessidade constante de reflexões sobre os pressupostos teóricos e procedimentos metodológicos aplicados a estudos cujos interesses sejam avaliar atitudes antinormativas, sobretudo, em contextos com características peculiares sobre as relações raciais, como é o caso do Brasil. Investigações mais amplas e atentas às sutilezas do fenômeno da desejabilidade social representam uma possibilidade de avanço no estudo das relações raciais. A infra-humanização aplicada ao racismo no contexto brasileiro ainda é um vasto caminho a ser explorado. 


\section{REFERÊNCIAS}

AGUIAR, P. \& LIMA, M. E. O. Estudo para validação da distinção entre traços de natureza e traços de cultura. In: M. E. O. Lima (2003). Normas sociais e racismo: efeitos do individualismo meritocrático e do igualitarismo na infra-humanização dos negros. Tese de doutorado não publicada, Instituto Superior de Ciências do Trabalho e da Empresa. Lisboa. Portugal, 2001.

BAIN, P. G. The structure and content of the human category, and its implications for understanding dehumanization. Humanness and dehumanization, 227-255, 2014.

BARASCH, A., LEVINE, E. E., BERMAN, J. Z., \& SMALL, D. A. Selfish or selfless? On the signal value of emotion in altruistic behavior. Journal of personality and social psychology, 107(3), 393, 2014.

BATSON, C. D. Two forms of perspective taking: imagining how another feels and imagining how you would fell. In: K. D. Markman, W. M. P. Klein \& J. A. Suhr. Handbook of imagination and mental simulation. New York: Psychology Press, 267279, 2009.

BATSON, C. D., KENNEDY, C. L., NORD, L., STOCKS, E. L., FLEMING, D. A., MARZETTE, C. M., LISHNER, D. A., HAYES, R. E. \& KOLCHINSKY, T. Z. Anger at unfairness: Is it moral outrage? European journal of social psychology, 37, 12721285, 2007.

BETTENCOURT, B. A., MANNING, M., MOLIX, L., SCHLEGEL, R., EIDELMAN, S., \& BIERNAT, M. Explaining extremity in evaluation of group members: Meta-analytic tests of three theories. Personality and Social Psychology Review, 20(1), 49-74, 2016.

CABECINHAS, R. Racismo e etnicidade em portugal: uma análise psicossociológica da homogeneização das minorias. Tese de doutorado. Universidade do Minho. Braga, 2002.

CAMINO, Leoncio et al. A face oculta do racismo no Brasil: Uma análise psicossociológica. Revista de psicologia política, v. 1, n. 1, p. 13-36, 2001.

CASTANO, E., \& GINER-SOROLLA, R. Not quite human: Infra-humanization as a response to collective responsibility for intergroup killing. Journal of Personality and Social Psychology, 90, 804-818, 2006. 
COSTA-LOPES, R., VALA, J., PEREIRA, C. \& AGUIAR, P. A construção social das diferenças nas relações entre grupos sociais. In: M. V. Cabral et al. (Eds.). Itinerários: A Investigação nos 25 Anos do ICS, Lisbon: Imprensa de Ciências Sociais, 769-790, 2008.

DASGUPTA, N. Mechanisms underlying the malleability of implicit prejudice and stereotypes. The role of automaticity and cognitive control. In: T. D. Nelson (Ed.), Handbook of prejudice, stereotyping, and discrimination. New York: Psychology Press, 61-87, 2009.

DASGUPTA, N. Implicit Ingroup Favoritism, Outgroup Favoritism, and Their Behavioral Manifestations. Social justice research, 17(2), 143-169, 2004.

DELGADO, N., BETANCOR, V., RODRÍGUEZ-PÉREZ, A., \& ARIÑO, E. The impact of helping behavior on outgroup infrahumanization. The Spanish journal of psychology, 15(3), 1099-1109, 2012.

DERKS, B., STEDEHOUDER, J., \& ITO, T. A. Social identity modifies face perception: an ERP study of social categorization. Social cognitive and affective neuroscience, 10(5), 672-679, 2014.

DESCHAMPS, J. C.; VALA, J.; MARINHO, C.; LOPES, R. C. \& CABECINHAS, R. Intergroup Relations, Racism and Attribution of Natural and Cultural Traits.

Psicología política, 30, 27-39, 2005.

EIDELMAN, S., SILVIA, P. J. \& BIERNAT, M. Responding to Deviance: Target Exclusion and Differential Devaluation. Personality and social psychology bulletin, 32, 1153-1164, 2006.

FERNANDES, SHEYLA C. S. Crenças raciais e infra-humanização: uma análise psicossocial do preconceito contra negros. Tese de Doutorado. Universidade Federal da Bahia. Salvador. Brasil, 2011.

FISKE, S. T., CUDDY, A. J. C. \& GLICK, P. S. (Dis)respect versus (dis)liking: status and interdependence predict ambivalent stereotypes of competence and warmth. Journal of social issues, 55, 473-489, 2009.

GAUNT, R., LEYENS, J. P., \& DEMOULIN, S. Intergroup relations and the attribution of emotions: Control over memory for secondary emotions associated with the in- 
group and outgroup. Journal of experimental social psychology, $38,508-514$, 2002.

HAIR, J. F. JR., ANDERSON, R. E., TATHAM, R. L. \& BLACK, W. C. Análise multivariada. Porto Alegre, RS: Bookman, 2006.

HAMMOND, R. A. \& AXELROD, R. The Evolution Of Ethnocentrism. Journal of conflict resolution, 50(6), 926-936, 2006.

HARVEY, Paul; MARTINKO, Mark J.; BORKOWSKI, Nancy. Justifying deviant behavior: The role of attributions and moral emotions. Journal of Business Ethics, v. 141, n. 4, p. 779-795, 2017.HASLAM, JETTEN, POSTMES \& HASLAM, 2009

HU, C. S., WANG, Q., HAN, T., WEARE, E., \& FU, G. Differential emotion attribution to neutral faces of own and other races. Cognition and Emotion, 31(2), 360-368, 2017.

KATZ, Daniel; BRALY, Kenneth. Racial stereotypes of one hundred college students. The Journal of Abnormal and Social Psychology, v. 28, n. 3, p. 280, 1933.

LEYENS, J., PALADINO, P., RODRIGUEZ-TORRES, R., VAES, J., DEMOULIN, S., RODRIGUEZ-PEREZ, A., \& GAUNT, R. The emotional side of prejudice: the attribution of secondary emotions to ingroups and outgroups. Personality and social psychology review, 4(2), 186-197, 2000.

LEYENS, J. PH., RODRIGUEZ-PEREZ, A., RODRIGUEZ-TORRES, R., GAUNT, R., PALADINO, P., VAES, J., \& DEMOULIN, S. Psychological essentialism and the differential attribution of uniquely human emotions to ingroups and outgroups.

European journal of social psychology, 31(4), 395-411, 2001.

LEYENS, J. P., \& VALA, J. "Back to the Future:" Ideological Dimensions of Intergroup Relations. In The Social Developmental Construction of Violence and Intergroup Conflict (pp. 85-104). Springer International Publishing, 2016.

LIMA, Marcus Eugênio Oliveira; VALA, Jorge. As novas formas de expressão do preconceito e do racismo. Estudos de psicologia (Natal), 2004.

LIMA, M. E. O., \& VALA, J. A cor do sucesso: Efeitos da performance social e econômica no branqueamento e na infra-humanização dos Negros no Brasil.

Psicologia USP, 16(3), 143-166, 2005. 
MARTÍNEZ, R., RODRIGUEZ-BAILON, R., MOYA, M., \& VAES, J. How do different humanness measures relate? Confronting the attribution of secondary emotions, human uniqueness, and human nature traits. The Journal of social psychology, 157(2), 165-180, 2017.

MOSCOVICI, S. \& PÉREZ, J. A. A extraordinária resistência das minorias à pressão das maiorias: o caso dos ciganos. In: J. Vala (Org.). Novos racismos: Perspectivas comparativas. Oeiras: Celta, 103-119, 1999.

MOSCOVICI, S. \& PÉREZ, J.A. Prejudice and social representations. Papers on social representations, 6(1), 27-36, 1997.

NOGUEIRA, O. Preconceito racial de marca e preconceito racial de origem Sugestão de um quadro de referência para a interpretação do material sobre relações raciais no Brasil. Tempo Social, revista de sociologia da USP, 19 (1), 287 308, 2007.

PALADINO P. M., LEYENS J. P., RODRIGUEZ R. T., RODRIGUEZ A. P., GAUNT R, ET AL. Differential associations of uniquely and nonuniquely human emotions with the in-group and the out-group. Group process and intergroup relations, 5,105-17, 2002.

RUTLAND, Adam; KILLEN, Melanie. A developmental science approach to reducing prejudice and social exclusion: Intergroup processes, social-cognitive development, and moral reasoning. Social Issues and Policy Review, v. 9, n. 1, p. 121-154, 2015.

RUTLAND, A., HITTI, A., MULVEY, K. L., ABRAMS, D., \& KILLEN, M. When does the in-group like the out-group? Bias among children as a function of group norms. Psychological Science, 26(6), 834-842, 2015.

TAJFEL, H. Grupos humanos e categorias sociais. Lisboa: Livros Horizonte, 1981.

TAYLOR, D. M., E GUIMOND, S. The belief theory of prejudice in an intergroup context. Journal of Social Psychology, 105, 11-25, 1978.

TURRA, C. \& VENTURI, G. Racismo cordial: a mais completa análise de preconceito de cor no Brasil. São Paulo. Ática, 1995. 
VALA, J, PEREIRA, C. \& COSTA-LOPES, R. Is the attribution of cultural differences to minorities an expression of racial prejudice? International journal of psychology, 44 (1), 20-28, 2009.

VALA, Jorge; BRITO, Rodrigo; LOPES, Diniz. Expressões dos racismos em Portugal. ICS. Imprensa de Ciências Sociais, 2015.

VALA, J. Racisms: Social representations, racial prejudice and normative pressures. Papers on Social Representations, 22, 1-29, 2013.

VILHENA, J. A violência da cor. Sobre racismo, alteridade e intolerância. Revista psicologia política. 6(12), 1-25, 2007.

WONDRA, J. D., \& ELLSWORTH, P. C. An appraisal theory of empathy and other vicarious emotional experiences. Psychological review, 122(3), 411, 2015.

YZERBYT, V. Y., CASTANO, E., LEYENS, J.-P., \& PALADINO, P. M. The primacy of the ingroup: The interplay of entitativity and identification. In W. Stroebe \& M.

Hewstone (Eds.). European review of social psychology. Chichester, England:

Wiley, 11, 258-295, 2000. 